\title{
Expertengespräch: Psychische Erkrankungen
}

Welches sind die häufigsten psychischen Störungen bei den Patienten, die Sie behandeln?

Albonico: Als Hausarzt und Spitalarzt überblicke ich das ambulante wie auch das stationäre Spektrum recht gut. Depressionen und Angststörungen sind weit im Vordergrund, assoziiert mit Erschöpfungszuständen, die man zunehmend auch Burnout nennt. Andere psychische Störungen sehe ich selten; Zwangsstörungen haben eher abgenommen, Phobien sind oft eine Definitionsfrage, bei der Posttraumatischen Belastungsstörung besteht ein grosses Problem der Begrifflichkeit jeder versteht darunter etwas anderes.

Dillner: Auch ich sehe in meiner Praxis vorwiegend Patienten mit Angststörungen und Depressionen bzw. Burnout, im Weiteren auch persönlichkeits- oder paarbezogene Problematiken. Ausserdem bin ich konsiliarisch tätig für das Paracelsus-Spital; dann stehen oft psychosomatische Probleme (z.B. Schmerzstörungen) oder seelische Reaktionen auf Krebserkrankungen im Vordergrund.

Flury: Die Hauptgruppe in der Klinik Schützen sind Depressionen bzw. Burnout, Angst-, Ess- und Persönlichkeitsstörungen sowie andere psychosomatische Störungen, z.B. psychische

Menschen mit einer psychischen Erkrankung profitieren am ehesten von einem ganzheitlichen Ansatz, der Körper und Seele, aber auch das soziale und kulturelle Umfeld miteinbezieht: Dies ist der gemeinsame Nenner des Expertengesprächs mit Hansueli Albonico, Chefarzt für interdisziplinäre Komplementärmedizin im Regionalspital Emmental, dem anthroposophischen Fachpsychiater Tomas Dillner von der Paracelsus-Klinik in Richterswil sowie dem Psychiater Hanspeter Flury, Chefarzt der Klinik Schützen in Rheinfelden.

Belastungsreaktionen auf somatische Erkrankungen wie Krebs (Psychoonkologie).

Gibt es Unterschiede zwischen Männern und Frauen? Andere Ursachen oder Krankheitsverläufe?

Flury: Zwei Drittel unserer Klientel sind Frauen. Es ist generell in der Psychiatrie und in der Medizin so, dass Frauen eher den Arzt aufsuchen. Für Männer ist die Schwelle höher, sich psychische Probleme einzugestehen, und sie haben auch einen anderen Umgang damit. Bei den Männern äussern sich diese eher auf der psychosozialen Ebene; sie haben Probleme am Arbeitsplatz oder kommen gar mit dem Gesetz in Konflikt. Männer suchen später Hilfe als Frauen - oft zu spät, wenn es rundum oder am eigenen Körper schon brennt.

Dillner: Das kann ich bestätigen. Vielleicht liegt darin ein Grund für die höhere Lebenserwartung der Frauen; zumindest wäre das für uns Ärzte eine erfreuliche Interpretation, aber so einfach ist es sicher nicht. In meiner Praxis behandle ich häufiger Frauen, aber die Verläufe sind in der Regel nicht anders als bei Männern. Statistisch gesehen gibt es in der Psychiatrie schon Häufigkeitsunterschiede zwi- schen den Geschlechtern: Zum Beispiel Alkoholismus eher bei Männern, psychosomatische Störungen eher bei Frauen.

Albonico: Depressionen und vor allem Burnout haben sehr viel zu tun mit der sozialen Einbindung, abhängig vom Rollenverständnis und der sozialen Situation, aber auch vom kulturellen Hintergrund. Und hier gibt es Unterschiede, die sich genderspezifisch äussern: Eine Asylantin z.B., die auf ihre Ausweisung wartet, hat rein vom äusseren Setting her Schwierigkeiten. Sie verfügt nicht über dieselben Ressourcen wie ein Mann. Deshalb gibt es auch unterschiedliche Verläufe.

Welche seelischen Erkrankungen lassen sich mit Ihrem Verfahren der Komplementärmedizin am besten behandeln?

Dillner: Generell können wir sagen, dass sich alle seelischen Erkrankungen in der anthroposophischen Medizin behandeln lassen. Das zeigt auch eine immer grössere Anzahl Studien. Gemäss dem anthroposophischen Menschenbild besteht der Mensch aus Geist, Seele und Körper. Schon Aristoteles war der Auffassung, dass die Seele gar nicht krank werden könne. Die Seele betrachtete er als formge-

\section{KARGER}

Fax +49761 4520714 


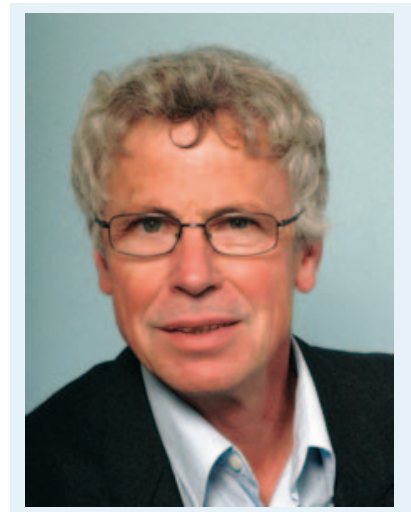

Dr. med. Hansueli Albonico

(hu.albonico@hotmail.com) ist anthroposophisch orientierter Hausarzt, Chefarzt der Komplementärmedizinischen Abteilung des Regionalspitals Emmental und seit 2009 Präsident der Union schweizerischer komplementärmedizinischer Ärzteorganisationen.

«Die AMOS-Studien zeigen: Der anthroposophische Ansatz bringt bei seelischen Erkrankungen mindestens gleich gute Resultate wie die Schulmedizin.»

bendes Prinzip, und eine Form kann nicht erkranken, nur in ihrer Entfaltung gestört werden. Sieht man es so, dann liegt die Ursache der seelischen Störung vielmehr im Körper.

Albonico: Im Rahmen der AMOS-Studien (Anthroposophic Medicine Outcome Study; siehe Kasten) wurden 2006 eine Depressionsstudie [1-3], 2009 eine Angststudie [4-5] und 2010 eine ADHS-Studie [6] publiziert, welche die Wirksamkeit, aber auch die Wirtschaftlichkeit der anthroposophischen Medizin für diese Indikationen gut belegen. Das sind bemerkenswerte Studien mit klaren Resultaten, einmalig im europäischen Raum, die uns in unserem täglichen Tun bestätigen. Man darf festhalten: Der anthroposophische Ansatz bringt mindestens gleich gute Resultate wie die sog. Schulmedizin.

Flury: Wir pflegen in der Klinik Schützen einen ganzheitlichen Ansatz. Das heisst: Wir wollen sowohl die psychische als auch die körperliche und soziale Situation, vielleicht sogar den
Menschen als sinnhaftes Wesen gesamthaft erfassen und in die Behandlung miteinbeziehen. Wir klären deshalb auch körperlich sehr gut ab, dann kommt die psychiatrisch-psychotherapeutische Abklärung und Therapie. Wir setzen individuell das ein, was nötig ist und am meisten erfolgversprechend in der jeweiligen Konstellation. Daneben kommen körpertherapeutische Methoden zur Anwendung Physiotherapie, Fitnessaufbau, Sport, Wassergymnastik, Körperausdruck und -wahrnehmung, Entspannungsverfahren wie autogenes Training oder progressive Muskelrelaxation. Auch Musik- und Maltherapie sowie Sozial- und Essberatung bieten wir an. Dazu kommt eine intensive Betreuung durch das Pflegefachpersonal.

\section{Was ist das Besondere an Ihrem Ansatz, seelische Erkrankungen zu behandeln?}

Flury: Es ist die Integration dieser verschiedenen Methoden, gekennzeichnet einerseits von dieser breiten Palette an sich, diewir individuell einsetzen. Andererseits die Therapieintensität, die wir in diesem offenen Rahmen bieten können; 3-6 Wochen so arbeiten, immer in der Absicht, die Symptome zu mildern, damit die Menschen selbstständig wieder besser zurechtkommen. Wir können nicht alles lösen, aber wir bieten einen Ansatz, der eine Trendwende ermöglichen soll, die nach Austritt im ambulanten Rahmen weitergehen soll. Unser Rahmen ist insofern speziell, als er das Gesunde, das Wohlbefinden betont; es ist eine relativ normale Umgebung mit gepflegter Hotellerie und der Nähe zum Städtchen. Man hat hier seine Therapien, wird als Mensch aber auch in seinen übrigen Dimensionen angesprochen. Das hat hier Platz.

Albonico: Die anthroposophische Medizin hat verschiedenste Ansatzpunkte, den Teufelskreis etwa einer Depression zu durchbrechen. Der Gegenpol zu Schwere und Dunkel ist Licht und Leichte. Dies finden wir zum einen bei den Heilmitteln, und das Stichwort hier heisst: Licht vermitteln. Gold (Aurum D30) spritzen wir bei Patienten mit Depressionen täglich. Licht lässt sich aber auch über den Stoffwechsel zuführen - hier nehmen wir eher Hepar-Magnesium; Magnesium ist ja auch so ein Lichtträger, denken Sie an die Wunderkerzen am Christbaum. Das spritzen wir regelmässig am Abend. Beim pflanzlichen Ansatz ist das Paradebeispiel natürlich $\mathrm{Hy}$ pericum (Johanniskraut). Es gibt aber in der Phytotherapie eine ganze Palette von Pflanzen und Arzneien, ebenso in der Homöopathie, die sich auf den einzelnen Patienten zugeschnitten anwenden lassen.

Für die Vermittlung von Licht und Leichte ist die anthroposophische Medizin zudem sehr geeignet mit der Rhythmischen Massage und mit der Heileurhythmie. Auch dies wird in der AMOS-Depressionsstudie bestätigt. Dann die künstlerischen Therapien - Malen, Plastizieren, Musik -, die dazukommen. Es geht ja darum, dass der Patient seine eigene Persönlichkeit wieder finden und durchsetzen kann; dafür sind diese Therapien hervorragend geeignet. Wir versuchen auch, aus der Biografiearbeit relevante Gesichtspunkte $\mathrm{zu}$ bekommen, um die Gesetzmässigkeiten krankhafter Entwicklungen zu eruieren, die häufig ihren Ursprung schon in der Kindheit und im Jugendalter haben. Es geht darum, dass der Patient diese Muster erkennen und «sein Leben in die Hand nehmen» lernt, wie es Gudrun Burkhard, die grosse Biografieautorin in der anthroposophischen Medizin, formuliert. Gerade im Spital sehe ich, dass dieses Thema grossen Anklang findet.

Wie steht es um eine schwere psychische Störung wie Borderline?

Dillner: Das ist ein gutes Beispiel für einen ganzheitlichen Therapieansatz, 


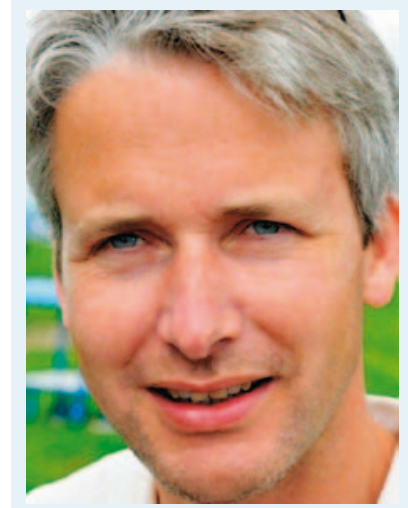

\section{Dr. med. Tomas Dillner}

(tomas.dillner@paracelsus-spital.ch) ist Facharzt für Psychiatrie und Psychotherapie mit eigener Praxis an der ParacelsusKlinik in Richterswil. Er stammt ursprünglich aus Norwegen, wo er auch seine fachärztliche Ausbildung absolvierte.

"Eine der Schwierigkeiten der Ganzheitsmedizin liegt darin, wenn nichtärztliche Therapeuten allein von sich aus behandeln. Es sollte zumindest ein Kontakt zu einem Psychiater bestehen.»

wie ihn die anthroposophische Medizin bietet. Man muss körperliche Übungen und künstlerische Therapien, Gesprächstherapie und die medikamentöse Behandlung integrieren. Gesprächstherapie, um das Ich zu wecken, damit, bildlich gesprochen, die Sonne wieder zum Scheinen kommt; Kunsttherapien, um das Gleichgewicht in der Seele zu schaffen; Heilmittel, um den Körper im Heilungsprozess zu unterstützen. Bei der Standardbehandlung sieht man nicht selten, dass Patientinnen mit Borderline-Diagnose - die Mehrheit der Betroffenen sind Frauen - am Schluss mit einer ganzen Reihe von Medikamenten dastehen: Antidepressiva, Antipsychotika, Antiepileptika, die sie zwar manchmal einigermassen stabil halten, aber zu einem hohen Preis; ich denke vor allem an die Sedierung. Eine solche Behandlung geschieht oft aus purer Not. Selbst dann ist eine anthroposophische Therapie noch möglich, und zwar im Sinne einer Erweiterung der schulmedizinischen Behandlung. Das langfristige Ziel besteht darin, die Psychopharmaka zu reduzieren, langsam eines ums andere. Ein langwieriger und zum Teil heikler Prozess.

\section{Wie können Psychiater und}

Komplementärmediziner für das Wohl der Patienten zusammenarbeiten?

Dillner: Ärztliche Komplementärmediziner arbeiten in der Regel unproblematisch mit Psychiatern zusammen. Aufpassen muss man bei nichtärztlichen Komplementärmedizinern, gerade bei psychischen Krankheiten. Es sollte zumindest ein Kontakt bestehen zu einem Psychiater. Meine Patienten haben oft Erfahrungen gemacht mit verschiedenen nichtärztlichen Komplementärmedizinern, bevor sie zu mir gekommen sind. In einem Fall konsultierte eine Patientin, die unter Panikattacken und Erschöpfung litt, eine Naturheilerin, die ihr «noch 25\% Lebensenergie in den Organen» attestierte. Ihre Angst wurde dadurch nicht gerade vermindert. In einem anderen Fall wurde einem Patienten empfohlen, zum Teil teure Schutzmassnahmen gegen «Elektrosmog» zu ergreifen.

Albonico: Diese Zusammenarbeit ist grundsätzlich wichtig. Es gibt ja auch einige anthroposophische Fachpsychiater. Wir versuchen natürlich, vor allem mit ihnen zusammenzuarbeiten. Erwähnen muss man an dieser Stelle, dass das schulpsychiatrische Spektrum unendlich breit ist. Es gibt eine starke Richtung, die sich auf Medikamente beschränkt, und andere, die nur psychoanalytische Methoden anwenden. Beides, finde ich, ist zu eng. Uns sind systemtherapeutisch orientierte Psychiater wichtig, welche die Familie und das soziale Umfeld miteinbeziehen.

Flury: Das interdisziplinäre Zusammenspiel ist in der Medizin ohnehin etwas total Wichtiges. Dazu gehört auch die Komplementärmedizin im Zusammenspiel mit der Schulmedizin -z.B. dass man sich und die jeweiligen
Behandlungsmethoden gegenseitig kennt. $\mathrm{Ob}$ man unsere Therapien in der Klinik Schützen komplementärmedizinisch nennen will? Eher nicht. Wir nennen sie «Spezialtherapien». Ich würde sie nicht bei den «grossen Fünf» der Komplementärmedizin ansiedeln, aber sie haben mit ihr insofern viel gemeinsam, als sie auf das Ganzheitliche zielen, in einem gesamtheitlichen Rahmen.

\section{Eignen sich Ihre Verfahren auch zur Behandlung von Kindern und Jugendlichen?}

Albonico: Sie sind sogar speziell geeignet, denn hier haben wir die doppelte Hemmung, starke Psychopharmaka einzusetzen. Erstens sind diese an Kindern wenig getestet, zweitens ist bei Kindern das ganze Nervensystem in Entwicklung. Ich persönlich bin deshalb auch mit Ritalin sehr zurückhaltend. Auch im Rahmen von AMOS konnte gezeigt werden, dass man in weitaus den meisten Fällen ohne Ritalin durchkommt.

Flury: Im Schützen behandeln wir keine Kinder und Jugendlichen. Im Übrigen finde ich aber, dass die ganzheitliche Sicht in dieser Altersgruppe besonders wichtig ist. Wie wollen Sie eine Schlafstörung eines Kindes nur vom Kind aus erklären? Hier muss man auch fragen, was läuft in der Peergroup, was in der Schule, was in der Familie, was entwicklungsmässig - bei Kindern und Jugendlichen braucht es fast immer ein gesamtheitliches Konzept.

\section{Wo liegen die grössten Schwierig- keiten bei der Behandlung von seelischen Erkrankungen mit Verfahren der Ganzheitsmedizin?}

Albonico: Grenzen soll man eingestehen, denn die gibt es, ganz klar: Schwere Psychosen - hier kommen wir ohne Psychiater und Psychophar- 


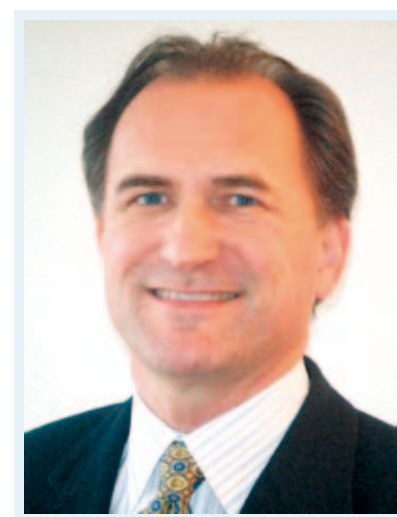

Dr. med. Hanspeter Flury

(hanspeter.flury@klinikschuetzen.ch) ist Chefarzt und Leiter der Klinik Schützen in Rheinfelden, einer Privatklinik für Psychosomatik und Psychotherapie. Er ist Facharzt für Psychiatrie und Psychotherapie.

"Unser Rahmen betont das Gesunde, das Wohlbefinden. Man hat hier seine Therapien, wird als Mensch aber auch in seinen übrigen Dimensionen angesprochen.»

maka nicht durch. Grenzen gibt es auch bei der hohen Suizidalität; gerade kürzlich musste ich einen jungen Mann einweisen, weil es gefährlich wurde. Grenzen gibt es auch bei langen Vorbehandlungen mit Allopathi$\mathrm{ka}$, wo es schwierig wird, mit anderen regulativen Einwirkungen einzugreifen, und zunehmend auch Grenzen durch das soziale Umfeld, Stichwort Burnout. Hier ist es oft unmöglich, Einfluss zu nehmen.

Dillner: Eine der Schwierigkeiten der Ganzheitsmedizin liegt wie gesagt darin, wenn nichtärztliche Therapeuten allein von sich aus behandeln. Genauso wenig, finde ich, können NichtPsychiater mit einem Zusatzkurs in Psychotherapie fachlich tragfähige Gesprächstherapie anbieten. Das reicht nicht. Man kann nicht, wie es in der psychiatrischen Facharztausbildung obligatorisch ist, auf die jahrelange Erfahrung in Akut-Abteilungen oder anderen stationären Abteilun- gen, auf die vielen Jahre mit Supervision und Selbsterfahrung einfach so verzichten. Das sind notwendige Erfahrungen, und sie sind nicht per Kurs vermittelbar.

Flury: Natürlich stossen wir an Grenzen im Bereich unserer offenen Institution. Beispielsweise können wir jemanden, der selbstmordgefährdet oder akut verwirrt ist, hier nicht aufnehmen. Spezialisierung ergibt Sinn, denn wenn man meint, man könne alles, wird es gefährlich.

\section{Wie muss ein ganzheitliches}

Behandlungskonzept aussehen?

Flury: Indem man versucht, den Menschen und seine Probleme, aber auch seine Ressourcen in ihrer Vielschichtigkeit zu erfassen, und dann individuell, d.h. nicht ideologiegetrieben, sondern in Kenntnis der zur Verfügung stehenden Möglichkeiten gemeinsam mit dem Patienten den Weg bestimmt.

Dillner: Das ist eine wesentliche und umfassende Frage. Das Wichtigste: Dem Menschen muss begegnet werden. Aus dieser Beziehung entsteht hoffentlich eine therapeutische Allianz, auf seelisch-geistiger Ebene.
Denn in der Gesprächstherapie wendet sich der Therapeut ja an das gesunde Ich des kranken Menschen, an seine Sonne sozusagen, um die Seele freizumachen. Und das bildet den Ausgangspunkt für alle anderen Massnahmen.

Albonico: Ganzheitlich heisst, z.B. bei unserer Asylantin: den Kontakt mit den Behörden aufzunehmen und $\mathrm{zu}$ erfragen, wie es denn um die Ausschaffung jetzt stehe. Das sind natürlich Luxustaten, die für ein Spital wirtschaftlich nicht mehr drinliegen werden. Aber die Erfahrung zeigt uns, wie wichtig dies wäre: Die Asylantin war unzählige Male hospitalisiert und kam dann zu uns; sie hatte sich völlig aufgeben, weil sie nur auf den Rückschaffungsbescheid wartete. Das tut sie immer noch, aber sie ist aufgeblüht und hat einen neuen Weg gefunden. Da kann man manchmal kleine Wunder erleben. Bei Depressionen ist wichtig: Das Umfeld miteinbeziehen. Und eine Prise Humor zu finden; das hält davon ab, in Zynismus und Sarkasmus und Suizid abzugleiten. Der Humor ist eine ganz spezielle Form von Geistesgegenwart, die über das Widerliche der Vergangenheit hinweggeht und Wege in die Zukunft findet.

Interview: Irène Dietschi

\section{AMOS-Studien}

1 Hamre HJ, et al.: Anthroposophische Therapie bei chronischer Depression: eine vierjährige prospektive Kohortenstudie. Der Merkurstab 2007;60:208-220.

2 Hamre HJ, et al.: Anthroposophic therapy for chronic depression: a four-year prospective cohort study. BMC Psychiatry 2006;6:57.

3 Hamre HJ, et al.: Health costs in patients treated for depression, in patients with depressive symptoms treated for another chronic disorder, and in non-depressed patients: a two-year prospective cohort study in anthroposophic outpatient settings. Eur J Health Econ 2010;11:7794.

4 Hamre HJ, et al.: Anthroposophische Therapie bei Angststörungen: eine zweijährige prospektive Kohortenstudie in der routinemässigen ambulanten Krankenversorgung. Der Merkurstab 2010;63:150-161.

5 Hamre HJ, et al.: Anthroposophic therapy for anxiety disorders: a two-year prospective cohort study in routine outpatient settings. Clinical Medicine: Psychiatry 2009;2:17-31.

6 Hamre HJ, et al.: Anthroposophic therapy for children with attention deficit hyperactivity symptoms: a two-year prospective cohort study in routine primary care settings. 2010 (filed for publication). 